\title{
LA CONTABILIDAD EN FUNCIÓN DE LA SUSTENTABILIDAD: UNA MIRADA DESDE EL DESARROLLO ECONÓMICO ALTERNATIVO
}

\author{
ACCOUNTING IN TERMS OF SUSTAINABILITY: \\ A VIEW FROM THE ALTERNATIVE ECONOMIC DEVELOPMENT \\ Eutimio Mejía Soto* \\ Docente de la Universidad del Quindío / Quindío - Colombia \\ Ciro Alfonso Serna Mendoza** \\ Docente de la Universidad de Manizales - Colombia \\ [Recepción: Setiembre de 2015 / Conformidad: Octubre de 2015]
}

\section{RESUMEN}

La contabilidad como ciencia se ha construido desde diversas concepciones epistemológicas, sociológicas, políticas y económicas; entre otros campos del saber, que ejercen influencia en el universo discursivo contable, prevaleciendo la visión amplia, holística, integradora y éticamente vinculada con el bienestar de la sociedad presente y futura. La regulación contable no se deriva necesariamente de la investigación; por ello, ha mantenido una tendencia reduccionista de este saber, al concebir a la contabilidad como el lenguaje de los negocios, sin más responsabilidad que la protección y el salvaguarde del interés de los proveedores de capital de riesgo. El presente trabajo pretende contribuir a la construcción teórica y al diseño práctico de una contabilidad para la sostenibilidad, entendida como un saber socio-ambientalmente responsable por su contribución a la protección de la riqueza ambiental, social y económica, a través de la evaluación de la gestión que las organizaciones realizan con respecto a las riquezas señaladas.

La contabilidad para la sostenibilidad se construye en diálogo con las ciencias naturales y las ciencias sociales. La biocontabilidad y la sociocontabilidad como disciplinas emergentes se orientan hacia una sustentabilidad ecológica y social, donde los aspectos económico-financieros deben estar al servicio de la naturaleza y de la sociedad.

Palabras clave:

Contabilidad; economía; naturaleza; sociedad; sustentabilidad.

\begin{abstract}
Accounting as a science has been built from various epistemological, sociological, political and economic concepts- among other fields of knowledge- that influence the accounting discursive universe, prevailing comprehensive, holistic, inclusive and ethical vision related to the welfare of present and future society. Accounting regulation is not necessarily derived from research; therefore, it has maintained a reductionist tendency of its knowledge, conceiving accounting as the language of business, with no more responsibility than the protection and safeguard the interest of venture capital providers.

This paper aims to contribute to the theoretical construction and practical design of an accounting for sustainability, understood as a knowlegde socio-environmentally responsible for contributing to the protection of the environmental, social and economic richness, through management assessment organizations made regarding the aforementioned richness.

Accounting for sustainability is built on dialoguing with the Natural Sciences and the Social Sciences. The bioaccounting and socioaccounting like other emerging disciplines, are oriented towards ecological and social sustainability, where economic and financial aspects should be at the service of nature and society.
\end{abstract}

Keywords:

Accounting; economy; nature; society; sustainability.

\footnotetext{
* Magister en Desarrollo Sostenible y Medio Ambiente - Universidad de Manizales. Contador Público y Filósofo - Universidad del Quindío. Email: eutimiomejia@uniquindio.edu.co

** Doctor en Ciencias Pedagógicas - Universidad de la Habana. Magíster en Desarrollo Social y Desarrollo Educativo - Universidad Pedagógica Nacional. Economista Universidad Pedagógica y Tecnológica de Colombia. Email: redesomciro@hotmail.com
} 


\section{INTRODUCCIÓN}

El presente documento tiene el objeto de explicar la necesidad de construir una nueva contabilidad, que permita el tránsito de la contabilidad monetaria a una contabilidad en otro tipo de unidades, tal consideración sustentada en la imposibilidad de la moneda de representary preservar todos los tipos de valores y riquezas existentes en la realidad. El tránsito de la contabilidad por partida doble a otros tipos de representación, y la finalidad de protección del capital económico hacia la protección de la riqueza ecológica es una urgencia sentida por la sociedad y conocida por la comunidad científica y profesional del saber contable. Georgescu-Roegen $(2011,189)$ se refiere a la diferencia que existe entre los sistemas de información empresarial en términos económicos y la realidad eco-sistémica en los siguientes términos:

Los economistas han intentado comunicar la idea de que normalmente por cada desembolso debe haber un ingreso equivalente. A largo plazo, los libros de cualquier empresa deben cuadrar, dólar a dólar...

Los libros de ecología nunca cuadran. No se llevan en dólares, sino en términos de materia-energía, y en estos términos siempre terminan con un déficit. De hecho, cada trabajo, de cualquier clase, hecho por un organismo vivo o por una máquina, se obtiene a un coste mayor del que ese trabajo representa en los mismos términos. Para poner un simple ejemplo, la energía aprovechable de una caldera de una máquina de vapor va en tres direcciones: una parte se convierte exactamente en el trabajo deseado de la máquina, una parte se disipa por el trabajo para vencer el rozamiento, y una parte es transferida al enfriador.

Los dos últimos elementos constituyen el déficit de la operación; la energía disipada por el razonamiento y la trasladada al enfriador ya no serán nunca más aprovechables por el hombre para obtener trabajo... Así pues, ésta es la cuarta ley de la ecología —e implícitamente, del proceso económico: hagamos lo que hagamos dará como resultado un déficit en términos de materia-energía”.

La contabilidad para la sostenibilidad debe tener la capacidad de articularse con la información en stocks y flujos de energía, de materiales y de información, sin abandonar la información monetaria, pero reconociendo el carácter subsidiario limitado de la misma. El resultado de la investigación muestra las reflexiones teórico-conceptuales desde concepciones económicas alternativas, que contribuyen a sustentar una contabilidad comprometida con un desarrollo duradero en términos naturales y sociales.

La contabilidad es una ciencia social, autónoma e independiente, que tiene relaciones de afinidad, formales, esenciales e instrumentales con otros campos del saber pero sin perder su esencia para realizar su función y alcanzar su fin social. Las dinámicas de la economía de mercado la han convertido en un instrumento al servicio del interés de la acumulación más que de la distribución.

La antigua separación, a veces incluso divorcio, entre el enfoque contable y financiero se ha terminado. Los criterios de evaluación financiera- y también bursátil en sentido estricto, se han impuesto a los contables. La contabilidad, bajo apariencias técnicas, "es de hecho un conjunto de construcciones sociales, históricamente fechadas y generadoras de efectos económicos" (Michael Capron). Esta victoria de la comunidad financiera y de los inversionista tomó varias décadas (Serfati, 2013, 10).

El presente artículo fundamenta que el propósito de lograr la sostenibilidad ambiental y social, requiere la contribución de la contabilidad como requisito necesario pero no suficiente para alcanzarla. La valoración cualitativa y cuantitativa de la existencia y circulación de la riqueza ambiental, social y económica es una necesidad apremiante en el proceso de construcción de acciones comprometidas con la naturaleza y la sociedad. El artículo desarrolla en su estructura, elementos que justifican la construcción de una contabilidad de la riqueza natural y social, sistemas denominados biocontabilidad y sociocontabilidad. El documento argumenta que la contabilidad económica es fundamental para la sociedad y las organizaciones, pero no es la única ni el más importante sistema de representación de la realidad, sin la información ambiental y social, cualquier información organizacional será siempre incompleta, sesgada y estéril como herramienta para la toma de decisiones. 


\section{MÉTODO}

El presente documento es resultado de una investigación de tipo exploratorio, por cuanto se pretende evaluar las posibilidades de sustentar un sistema contable ambiental sustentado en corrientes económicas diferentes a la economía neoclásica y específicamente distante del modelo económico neoliberal. El trabajo desarrollado utilizó el método deductivo, identifica los principios generales de las propuestas teóricas de economías alternativas y formula las razones que motivan su aplicación en un campo específico como es la biocontabilidad. El documento tiene un carácter propositivo una vez que presenta y sustenta la propuesta de un nuevo sistema contable como es la biocontabilidad, como un proceso de preparación y presentación de información contable de la naturaleza en unidades no monetarias, sustentada en la finalidad de alcanzar la sustentabilidad de la riqueza natural y la satisfacción de las necesidades de los hombres de manera responsable con otras especies vivas y las futuras generaciones.

\section{RESULTADOS Y DISCUSIÓN}

\section{La contabilidad como un saber para la sustentabilidad}

La Teoría Tridimensional de la Contabilidad T3C considera que la contabilidad económica ${ }^{1}$ es solo una de las aristas del saber contable. Las otras dos aristas son la biocontabilidad y la sociocontabilidad, definidas como disciplinas científicas encargadas de evaluar la gestión de la organización en el control de la riqueza ambiental y social, en función de la importancia de la naturaleza y la sociedad, en el marco de una fundamentación biocéntrica -o ecocéntrica- de la contabilidad, apartada de los cálculos exclusivamente rentísticos y financieros. Los estudios ontológicos, epistemológicos, axiológicos, teleológicos e históricos de la contabilidad señalan que es una ciencia autónoma, con un objeto que supera los límites de la realidad económico-financiera a los que la ha confinado la regulación económica en las diferentes jurisdicciones incluyendo la normalización internacional.
La riqueza ambiental y social no cuenta con instrumentos contables para velar por la protección y conservación de la misma, en tal sentido se hace necesario el desarrollo de sistemas contables orientados a cubrir la necesidad de evaluar la gestión de la organización que ejerce sobre la riqueza natural y social con fines de lograr su cuidado y protección. La biocontabilidad y la sociocontabilidad pretenden hacer parte de la respuesta a la mencionada e inaplazable necesidad. La contabilidad (financiera) ambiental yla contabilidad (financiera) social se han diseñado para acrecentar las utilidades del capital, en tal sentido no han respondido a la sustentabilidad de dicha riqueza.

La nueva contabilidad se relaciona y se complementa con la ecología, la economía, la historia, la sociología, la psicología y todas las ciencias, se nutre fundamentalmente, de los discursos humanistas y naturalistas, que desde posiciones éticas bio-incluyentes elevan la responsabilidad del hombre a todas las formas de vida y las generaciones del porvenir. La sustentabilidad es una finalidad de las diversas áreas del saber, propósito en el cual convergen las ciencias naturales y sociales, para develar los factores que hacen del modelo de desarrollo un riesgo permanente para alcanzar tan anhelado propósito.

Las Naciones Unidas $(1987,59)$ definen:

El desarrollo duradero [sostenible] es el desarrollo que satisface las necesidades de la generación presente, sin comprometer la capacidad de las generaciones futuras para satisfacer sus propias necesidades. Encierra en sí dos conceptos fundamentales:

1. El concepto de "necesidades", en particular las necesidades esenciales de los pobres, a las que se debería otorgar prioridad preponderante;

2. La idea de limitaciones impuestas por la capacidad del medio ambiente para satisfacer las necesidades presentes y futuras.

Carrasco $(2010 ; 34,35)$ construye una nueva conceptualización del término desarrollo sostenible, al afirmar que:

El desarrollo sostenible es un proceso en que la explotación de recursos, las direcciones de inversiones y los cambios institucionales son todos hechos consistentes con un futuro común tejido como necesidad del presente. El concepto de desarrollo sostenible

1 La contabilidad financiera es una aplicación dentro de la contabilidad económica. 
tiene el subordinado que establece como premisas los siguientes puntos:

1. Relación simbiótica entre el consumidor (la raza humana) y el productor (el sistema natural).

2. Compatibilidad entre la ecología y la economía.

La sustentabilidad no es un concepto omnímodo, por el contrario es un término polisémico, por lo tanto, se hace necesario clasificar las diferentes acepciones del concepto en grandes categorías, Ramírez, Sánchez y García (2004; 57, 58) clasifican la sustentabilidad en cinco categorías: ecologista, intergeneracional, sectorial, económico y enfoque de gestión.

La polémica entre sostenibilidad débil y sostenibilidad fuerte tiene diversas aristas, que hacen tránsito desde posturas teóricas, hasta diseños técnicos que intentan fundamentar la validez de sus postulados. Los supuestos de la ecología profunda contribuyen no solo a discernir la dicotomía entre posiciones extremas, sino que además, constituyen referentes válidos para la construcción de un corpus teórico de una contabilidad comprometida con la sustentabilidad en un sentido fuerte. Arne Naess (citado por García, 2005, 105), presenta los siguientes ítems característicos de esta disciplina:

1. Rechazo y sustitución de la imagen del "hombreen-el-medio (entorno)" por la (imagen) de "campo total" o "relacional".
2. Igualitarismo biosférico como criterio general.

3. Principios de diversidad y simbiosis. La diversidad incrementa las posibilidades de supervivencia, las oportunidades para nuevas formas de vida y la riqueza de formas de vida.

4. Postura anti-clase.

5. Lucha contra la contaminación y el agotamiento de los recursos.

6. Complejidad no complicación. El hombre desconoce las relaciones biosféricas por eso las complica, sin entender su complejidad.

7. Autonomía local y descentralización.

La contabilidad como saber estratégico al servicio de la transformación social, se desarrolla teóricamente desde una o varias de estas consideraciones en torno a la sostenibilidad débil o fuerte y sus puntos intermedios. Las últimas décadas las orientaciones contables relacionadas con la naturaleza, han tenido como referente la economía ambiental; desde el presente documento se propone la economía ecológica como uno y no el único referente, orientación complementaria de constructos de ecología, biología, sociología, antropología y otras áreas del saber que permitirán construir un saber contable comprometido con las grandes transformaciones que la sociedad reclama y las generaciones futuras merecen desde una visión ética con responsabilidad intra e intergeneracional. 


\section{Cuadro No 01}

\section{Esquema diferencia entre sustentabilidad débil y sustentabilidad fuerte}

\begin{tabular}{|l|l|}
\hline \multicolumn{1}{|c|}{ Sustentabilidad débil } & \multicolumn{1}{|c|}{ Sustentabilidad fuerte } \\
\hline $\begin{array}{l}\text { Visión antropocéntrica con énfasis en lo económico o o } \\
\text { social. }\end{array}$ & $\begin{array}{l}\text { Visión biocéntrica (énfasis en la naturaleza con igual- } \\
\text { dad biótica-ecología profunda) y concepción eco-cén- } \\
\text { trica (respeto del hombre a las leyes de la naturaleza). }\end{array}$ \\
\hline $\begin{array}{l}\text { Sostenibilidad económica y social del modelo de } \\
\text { desarrollo. }\end{array}$ & Sostenibilidad ecológica de la forma de vida. \\
\hline $\begin{array}{l}\text { Los recursos son insumos o materias primas para satis- } \\
\text { facer las necesidades humanas. }\end{array}$ & $\begin{array}{l}\text { Los recursos existen en condiciones diversas para } \\
\text { garantizar la estabilidad de las relaciones ecosistémicas. }\end{array}$ \\
\hline $\begin{array}{l}\text { Evalúa la función y existencia de recursos en el corto } \\
\text { plazo. }\end{array}$ & $\begin{array}{l}\text { Evalúa la función, finalidad y existencia de los bienes } \\
\text { ambientales en el largo plazo. }\end{array}$ \\
\hline $\begin{array}{l}\text { Evaluación focalizada en un tiempo y un espacio de los } \\
\text { impactos ambientales. }\end{array}$ & $\begin{array}{l}\text { Evaluación holística en el tiempo y en el espacio de las } \\
\text { acciones de los hombres sobre el ambiente. }\end{array}$ \\
\hline Perspectiva monetaria. & Perspectiva de valoración ecología. \\
\hline $\begin{array}{l}\text { Considera que los recursos son ilimitados o por lo } \\
\text { menos sustituibles. }\end{array}$ & Considera los recursos finitos y limitados. \\
\hline Fundamentada en la ciencia económica. & Fundamentada en la ecología y las ciencias naturales. \\
\hline $\begin{array}{l}\text { Relaciones de dominación y explotación } \\
\text { hombre/naturaleza. }\end{array}$ & Relaciones de mutua colaboración y respeto. \\
\hline $\begin{array}{l}\text { Confianza en la tecnología para mejorar la productivi- } \\
\text { dad y para corregir deterioros (optimismo tecnológico). }\end{array}$ & $\begin{array}{l}\text { Considera la existencia de daños irreversible de las con- } \\
\text { diciones naturales (pesimismo tecnológico). }\end{array}$ \\
\hline El desarrollo como finalidad. & La sustentabilidad como finalidad. \\
\hline Perspectiva de crecimiento ilimitado. & Perspectiva de crecimiento armónico. \\
\hline $\begin{array}{l}\text { Los bienes artificiales pueden reemplazar los bienes na- } \\
\text { turales. }\end{array}$ & $\begin{array}{l}\text { Los bienes naturales son insustituibles en sus funciones } \\
\text { ecosistémicas. }\end{array}$ \\
\hline
\end{tabular}

Fuente: Ramírez, Sánchez y García (2004) y Carrasco (2010).

Elaboración propia. 
La contabilidad debe reconocer que la satisfacción de las necesidades de las generaciones presentes y futuras de las especies vivas, se sustentan en recursos finitos, en términos de Naredo (1998, citado por Carrasco, 2010, 102) "La tierra es un sistema abierto en energía pero cerrado en materiales; es decir, donde es más fácil convertir materiales en energía, que energía en materiales". La economía por lo tanto debe comprenderse como un subsistema abierto, que implica que sus acciones tienen impactos en otros subsistemas. La tierra que es un sistema mayor, es un sistema cerrado en términos de recursos que proveen a las especies vivas la posibilidad de supervivencia en el tiempo como ecosistemas y como especies. Carrasco $(2010,102)$ advierte "la necesidad de hacer un planteamiento más próximo a la sostenibilidad; preservar los recursos y los límites a los cuales (la naturaleza) puede llegar".

La Teoría Tridimensional de la Contabilidad T3C, desarrolla tres disciplinas ${ }^{2}$ : biocontabilidad, sociocontabilidad y contabilidad económica, a continuación se explica cada una de ellas:

\section{1.- La biocontabilidad una respuesta sustentable a la contabilidad ambiental empresarial}

La propuesta de inclusión de la naturaleza en los estados e informes contables es un hecho de reciente aparición. La década del setenta presenta sus primeros atisbos, pero es posterior a la Declaración Río en 1992 y la Agenda 21 cuando el tema naturaleza-ambiente hará parte del quehacer contable.

La contabilidad ambiental, derivada de la economía ambiental es una herramienta que desde visiones antropocentrista y economicista otorga un valor monetario a los denominados "bienes y servicios ambientales o ecosistémicos" criterio sustentado en los beneficios económicos o asimilables que dichos bienes o servicios representan para el hombre. Gutiérrez y González $(2012,22)$ señalan que: "Esta reducción del ambiente a un conjunto de recursos a ser explota- dos para fines económicos remite, a una concepción de valor instrumental en la que el ambiente es útil sólo en la medida que satisface necesidades humanas, sin considerar a los otros seres vivos."

La primera década del siglo XXI, ha dado luz a nuevas visiones de la relación contabilidad y naturaleza. Se presentan diversos argumentos en oposición a la contabilidad ambiental, asimismo, se han presentado nuevas propuestas alternativas tales como la contabilidad ecológica cultural (Quinche, 2009), contabilidad socio-ambiental (Carbal, 2011) Sistema de contabilidad ambiental y económica SCAE (Naciones Unidas, 2012), contabilidad eco-ambiental (Leite y Dauzacker, 2010), sistema de cuentas de control y balanza ambiental "Sccobamb" (Mantilla, 2006); econtabilidad (Caro, 2011) y la biocontabilidad (Mejía, Montes y Mora, 2013), entre otros.

La explotación de los recursos naturales sin evaluación de sus límites y capacidad de aprovechamiento fue una constante en la historia económica de las naciones. La inclusión de la temática ambiental en las reuniones multilaterales fue una reacción de la economía de mercado al inminente riesgo que para sus intereses representaba el agotamiento constante y generalizado de los recursos naturales, los cuales son claves e imprescindibles en la dinámica extractiva propia de la relación entre las potencias económicas y los países de la periferia.

Martínez y Roca $(2013,253)$ afirman que:

La economía ecológica pone en duda el supuesto habitual de la teoría económica del crecimiento de que la inversión actual lleva a que las generaciones futuras serán más ricas. Puede ser que su creciente riqueza esté mal medida al basarse en la destrucción de recursos y servicios ambientales. Según la economía ecológica, la riqueza media de las generaciones futuras tal vez será inferior a la de la generación actual, dado el agotamiento de recursos naturales, los cambios climáticos globales y los límites a la sustituibilidad de materiales.

2 El término disciplina, debe entenderse como un abordaje temático especializado al interior de una ciencia, en el caso de la bio-contabilidad consiste en una hibridación disciplinar al articular diversas disciplinas para afrontar un problema de la realidad, genera una ruptura con la concepción clásica de disciplina en relación con un objeto específico estudiado por un campo del conocimiento en particular; la concepción utilizada refiere a saberes de las ciencias sociales y de las ciencias naturales que confluyen en un objeto-problema para articular conjuntamente su estudio descriptivo-explicativo propio del deber ser, para posteriormente generar una intervención del mismo fundamentada en criterios ético, teleológicos y estratégicos en el marco del deber ser; relación similar en correlación con otros campos del saber se presenta con la sociocontabilidad y la contabilidad económica. 
La biocontabilidad es una disciplina que evalúa la gestión de la organización en el control de la riqueza ambiental. Evaluación que permitirá adoptar decisiones tendientes a la sostenibilidad de la naturaleza y las relaciones eco-sistémicas. La biocontabilidad es una de las tres aristas de los sistemas de información contable, que en conjunto con la información contable social (sociocontabilidad) y la información contable económica (contabilidad económica) presentan la información suficiente de la organización y el tratamiento que otorga a la riqueza que la sociedad le ha confiado. La lectura integral del aporte a la sostenibilidad de la organización se logra a partir de la lectura conjunta de los tres juegos de estados contables: los ambientales, los sociales y los económicos.

\section{2.- Sociocontabilidad una alternativa a la conta- bilidad social}

Las organizaciones entendidas como células sociales, sistémicas y dinámicas generan impactos en las dimensiones ambiental, social y económica como resultado de sus acciones y omisiones. Los impactos que las organizaciones generan en relación con la acumulación, generación, distribución, consumo y sostenibilidad de las riquezas debe ser informada en los estados e informes contables. Las nuevas dinámicas públicas, las relaciones sociales y el avance de la legislación han llevado a que las organizaciones amplíen los actores que vigilan su actuación, el número de usuarios de la información y la relación con la sociedad en general. La rendición de cuentas como medio de comunicación empresa-sociedad-Estado tiene en la contabilidad el mejor instrumento de control social para garantizar la protección y cuidado en términos de tiempo y espacio de la riqueza ambiental, social y económica que la sociedad le ha confiado para ser gestionada en función del interés general.

La hegemonía de la visión económica sobre las otras dimensiones de la realidad, han generado importantes problemas sociales, tales como: el analfabetismo, la pobreza extrema, las zonas híper-degradadas, la explotación y expropiación de la riqueza de los países periféricos por parte de las potencias económicas, los conflictos bélicos, las guerras por los recursos naturales, la contaminación, la pérdida de autonomía política, alimentaria y económica de las naciones, son apenas un pequeño reflejo de las consecuencias de un afán desmedido de lucro por parte de los hombres, en gran medida representados en organizaciones públicas y privadas.

La sociocontabilidad es una propuesta enmarcada en la visión sustentable de las ciencias, se diferencia de la contabilidad social, porque mientras la primera tiene como fin la sustentabilidad y protección de las riquezas sociales, la segunda centra su interés en la identificación de los beneficios que para la organización y sus interés económicos representa la riqueza social.

La Socio-contabilidad es la disciplina que estudia la valoración cualitativa y cuantitativa de la existencia y circulación de la riqueza social controlada por las organizaciones, utilizando diversos métodos que le permiten evaluar la gestión que la organización ejerce sobre la mencionada riqueza social, con el fin de contribuir a la acumulación, generación, distribución y sostenibilidad integral de la misma (riqueza social). (Mejía, Montes y Mora, 2013, 47).

La necesidad de conocer las consecuencias que para la sociedad tienen las acciones de las organizaciones, más allá de internalizar externalidades, se debe reconocer en valores sociales más que en valores económicos dichos impactos, concepción que constituye la principal razón del por qué la sociocontabilidad debe implementarse en las organizaciones, para hacer seguimiento a la conducta organizacional y adoptar medidas tendientes a promover los comportamientos que generan beneficios a la sociedad, impedir los actos que afectan los valores y la riqueza social y en los casos donde el daño social se haya causado, penalizar a las infractoras como medio de disuasión para la transgresora y para que otras organizaciones no actúen en tal dirección; además, debe repararse a quienes sufrieron las consecuencias de una conducta no responsable de la organización.

La sociocontabilidad permite hacer pública, transparente y visible la información de los impactos sociales de la organización, ello constituye un control social y autocontrol organizacional frente al deber ser moral de las células sociales, velar por el interés público y el bien común. 


\section{3.- La contabilidad económica visión alternativa de la contabilidad financiarizada}

La economía debe ser una rama de la biología interpretada de forma amplia, descansa en el nivel más elemental de la cuestión. Somos una de las especies biológicas de este planeta, y como tal estamos sometidos a todas las leyes que gobiernan la existencia de la vida terrestre. Efectivamente somos una [especie] única, pero no porque hayamos obtenido el control total sobre los recursos de nuestra existencia. Los que piensan así nunca han comparado nuestra propia lucha por la existencia con la de otras especies, la de la ameba si deseamos un buen caso de análisis. No podemos estar seguros de que para un intelecto imparcial de otro mundo, que estudiara la vida terrestre tal y como un biólogo estudia el mundo de los micro-organismos (por ejemplo), la ameba no apareciese como una forma de vida con más éxito (Georgescu-Roegen, 2011, 194).

La contabilidad nacional o macrocontabilidad además de estar sometida a las críticas que advierten que es estadística o econometría pero no contabilidad, debe responder a los interrogantes que desde concepciones alternativas a la economía de mercado se presentan, tal como lo señala Daly $(2012,52)$ en la crítica al cálculo del Producto Interno Bruto, objeción que se suma a una pléyade de lineamientos en la misma dirección:

Una Economía en Estado Estacionario no debería contar con un sistema nacional de cuenta de resultados -el PIB- de la cual jamás se resta nada. Lo ideal sería tener dos cuentas, una que mida a escala los beneficios derivados del crecimiento físico, y otra que mida los costes de dicho crecimiento. Las políticas deberían basarse en frenar el crecimiento cuando los costes marginales sean iguales a los beneficios marginales. $\mathrm{O}$, si lo que queremos es mantener un único concepto de cuenta de resultados, deberíamos adoptar el concepto de renta del economista premio nobel, J. R. Hicks, a saber, la cantidad máxima anual que puede consumir una comunidad, sin dejar de ser capaz de producir y consumir la misma cantidad el siguiente año. Es decir, la renta es la cantidad máxima que puede llegar a consumirse sin perder capacidad productiva (capital). Todo consumo de capital, ya sea artificial o natural, deberá restarse a la hora de calcular la renta. Debemos de abandonar la práctica asimétrica de sumar al PIB la producción de anti-nocivos sin ha- ber restado primero la generación de dichos nocivos que precisamente requirieron anti nocivos.

El modelo de internacionalización de la contaduría profesional a través de la inclusión en la Arquitectura Financiera Internacional AFIC de los estándares contables y los estándares de auditoría, evidencia el interés que la profesión tiene para el gran capital financiero internacional. Diversas críticas se han formulado frente al modelo de desarrollo de los mercados financieros, el cual no centra su atención en la obtención de la ganancia a través del trabajo, no tiene interés en la generación de empleo, ni se preocupa por la satisfacción de necesidades de la población; su propósito primordial es la apropiación y acumulación de riqueza a través del flujo de capital especulativo. Serfati $(2013,7)$ explica el avance de los mercados financieros en los siguientes términos:

El capital financiero reposa sobre una función específica del dinero, su capacidad para producir más dinero, en la medida en que surge como capital ya sea bajo la forma de crédito bancario o bono o de la compra de un título de propiedad (acciones y derechos de propiedad intelectual). En suma, el capital financiero designa la capacidad del dinero para producir dinero...

La crítica debe formularse en referencia al funcionamiento improductivo del capital, donde el dinero genera dinero, sin producir bienes y servicios para satisfacer las necesidades sociales. El capital financiero empresarial se asocia al trabajo, a la producción, comercialización y consumo; por el contrario, la financiarización es una etapa nociva y una degradación de la actividad productiva socialmente útil, que convierte los mercados en un flujo de títulos que generan usurpación de riqueza sin la medicación de la producción real. Álvarez y Luengo $(2011,127)$ presentan la siguiente explicación del término financiarización:

El concepto de financiarización, de reciente generalización en la literatura económica, ha sido utilizado para referirse - de una u otra forma- al creciente dominio que los mercados y la lógica financiera ejercen sobre el conjunto de la dinámica económica desde finales de la década de 1970 ... algunos autores han utilizado este término para señalar el vertiginoso proceso de liberalización de los mercados financieros internacionales, la creciente inestabilidad de los mercados de 
divisas, la desintermediación y mercantilización de los sistemas financieros tradicionalmente centrados en torno a la banca, la formación de enormes burbujas bursátiles y crediticias, así como el impacto que todo ello tiene en el funcionamiento macroeconómico de las diversas economías nacionales.

Serfati $(2013,5)$ sobre el alcance del sector financiero señala que "las empresas transnacionales pueden definirse como grupos financieros con actividades industriales". Corrientes alternativas proponen otras formas de desarrollo distintas a las que han caracterizado la propuesta neoliberal. Daly (2012, 44) sustenta la posibilidad de alcanzar un desarrollo sin crecimiento, afirma que "el llamado crecimiento "económico" ya es antieconómico. El crecimiento económico está fracasando. Es decir, la expansión cuantitativa del subsistema económico provoca un incremento de los costes medioambientales y sociales a un ritmo mayor que el de la rentabilidad económica”.

Diversos autores presentan propuestas alternativas a la economía de mercado o visiones de mercado pero con matices de tipo socio-ambiental, entre ellas cabe destacar, la economía ambiental (Azqueta, 1998), el desarrollo a escala humana (Max Neef, 2000), economía ecológica (Martínez Alier y Rocca, 2013), la bioeconomía (Georgescu-Roegen, 2007), la economía de estado estacionario (Daly, 2012), la economía verde (Michael Jabobs), estructuralismo y neo-estructuralismo (Cepal), Serfati (2013), economía política ambiental (neo-marxistas-Torres, 2015), la economía del bien común (Felber).

El panorama teórico, conceptual y práctico de la economía tanto positiva como normativa, evidencia la ausencia de un acuerdo de general aceptación frente al ser y deber ser de la misma, en consecuencia, la contabilidad no puede construirse desde una sola visión de la economía, debe asumir el saber contable una independencia crítica tanto de las escuelas económicas, como de la economía misma y fundamentarse en todas las ramas del saber, de forma tal que pueda cumplir su función y finalidad asociada con la sostenibilidad de las riquezas naturales, sociales y económicas.

\section{CONCLUSIONES}

1. La contabilidad es la ciencia social aplicada que estudia la valoración cualitativa y cuantitativa de la circulación y existencia de la riqueza ambiental, social y económica que controlan las organizaciones, utilizando diversos métodos que le permiten cumplir su función de evaluar la gestión que la organización ejerce sobre la riqueza mencionada, con el fin de contribuir a la óptima acumulación, generación, distribución y sustentabilidad de las misma [riqueza] ${ }^{3}$.

2. La contabilidad es un saber interrelacionado con las ciencias formales, sociales y naturales, se complementa y complementa otros campos del saber.

3. La contabilidad actualmente está sometida al universo discursivo de la economía neoliberal.

4. La contabilidad debe utilizar unidades de medida diferentes a la moneda para el reconocimiento, presentación y revelación de información contable de la riqueza ambiental, social y económica. La dimensión ambiental y social requieren su representación en nuevas múltiples unidades de medida, como pueden ser las unidades de valor ambiental UVA y las unidades de valor social UVS.

5. La evaluación integral de la gestión de la organización, exige el análisis holístico del comportamiento de la riqueza ambiental, social y económica, para lo cual, deberán presentarse los tres tipos de estados contables en las dimensiones señaladas e integrarlos en un estado contable tridimensional [incluso multidimensional]. La presentación de información por dimensiones corresponde a una abstracción teórico-técnica, la realidad se comporta de manera sistémica, holística e integrada; un movimiento en una dimensión implica movimientos en las otras.

6. La contabilidad ha sido instrumentalizada conforme a la lógica de la financiarización, se encuentra al servicio del proceso de acumulación de riqueza económica a cualquier costo ambiental y social. El presente documento propone una ruptura paradigmática de la contabilidad, un repensar de su 
orientación ética, de forma que asuma su estatus de cientificidad, su función y finalidad de protectora del interés general, una vez que la sociedad históricamente le ha encomendado la misión de ser guardián de la riqueza para el bien común, en beneficio de la presente y las futuras generaciones de formas y expresiones de vida.

\section{REFERENCIAS BIBLIOGRÁFICAS}

1. ÁlVAREZ, I. \& LUENGO, F. (2011) Financiarización, acumulación de capital y crecimiento salarial en la UE-15. Investigación económica, vol. LXX, 276, abril-junio, pp. 125-162.

2. AZQUETA, D. Valoración económica de la calidad ambiental. Madrid: McGraw-Hill.

3. CARBAL,A. (2011) Una redefinición de la contabilidad socio-ambiental a partir del paradigma de la complejidad: consideraciones básicas. Revista Lúmina No 12, 280-299.

4. CARO, J. (2011) Contabilidad ambiental: las ventajas de un desarrollo sustentable. Buenos Aires: Edición.

5. CARRASCO, R. (2010) La sustentabilidad y las ideas... oníricas. Madrid: LASUR.

6. DALY, H. (2012) Una economía de estado estacionario. Papeles de relaciones eco-sociales y cambio global. No 117, pp. 43-55.

7. GARCÍA, M. (2005) Ecología profunda y educación. Tesis doctoral, Universidad Complutense de Madrid.

8. GEORGESCU-ROEGEN, N. (2007) Ensayos bio-económicos. Madrid: Catarata.

9. GEORGESCU-ROEGEN,N. (2011) ¿Quépuede enseñar a los economistas la termodinámica y la biología? En: Aguilera Klink, Federico y Alcántara, Vicent. De la economía ambiental a la economía ecológica. Madrid: FUHEM.

10.GUTIÉRREZ, E. \& GONZÁLEZ, E. (2012) De las teorías del desarrollo al desarrollo sustentable. Ciudad de México: Siglo XXI editores-UANL.

11.MANTILLA, E. (2006) La contabilidad en el desarrollo sostenible. Revista Legis Internacional de Contabilidad y Auditoría. No 25, enero-marzo, pp. 133-160.
12.MARTÍNEZ A, J. \& ROCA, J. (2013) Economía ecológica y política ambiental. México: FCE.

13. MAX, M.Et al. (2000) Desarrollo a escala humana: una opción para el futuro. Medellín: Proyecto 20 editores.

14. MEJÍA, E., MONTES, C. \& MORA, G. Estructura conceptual de la Teoría Tridimensional de la Contabilidad. Contexto, Vol. (2), 34-53.

15. Naciones Unidas (2012) Sistema de contabilidad ambiental y económica (SCAE) Marco Central. Ginebra. Naciones Unidas.

16. Naciones Unidas (1987) Informe de la Comisión Mundial sobre el Medio Ambiente y el Desarrollo "Nuestro futuro común". Naciones Unidas.

17.PEREIRA, F. \& DAUZACKER, N. (2010) Contabilidad Eco-ambiental. Registro de los efectos externos - un enfoque en los impactos ambientales y obtención del PEEB - Producto Ecológico Empresarial Bruto. Revista "Contabilidad y Auditoría” No 31. Pp. 14q-162.

18. POGGE, T. (2014) Crítica al progreso cosmético de la pobreza y el hambre del Banco Mundial ylos objetivos del milenio. Mundo Siglo XXI, No 34, Vol. X, pp. 5-22.

19.QUINCHE, F. (2009) Contabilidad y naturaleza: apuntes para una discusión. Libre Empresa No 12, 65-75.

20. RAMÍREZ, A.; SÁNCHEZ, J. \& GARCÍA, A. (2004) El desarrollo sustentable: interpretación y análisis. Revista del Centro de Investigación. Universidad de la Salle (México), vol. 6, No 21, pp. 55-59.

21. RITTERMAN, J. (2015) América Latina y Monsanto. Mundo siglo XXI, No 35, Vol. X, pp. 5-20

22. SERFATI, C. (2013) La lógica financiera-rentista de las sociedades transnacionales. Mundo Siglo XXI. Vol. VIII, No 29, pp. 5-21.

23. TORRES, G. (2015) La economía política ecológica. Seminario en tendencias económicas. Escuela Superior de Economía ESE. Instituto Politécnico Nacional IPN. Febrero-marzo, Ciudad de México. 